CIENCIAMATRIA

Revista Interdisciplinaria de Humanidades, Educación, Ciencia y Tecnología

Año V. Vol. V. N09. Julio - Diciembre 2019

Hecho el depósito de ley: pp201602FA4721

ISSN-L: 2542-3029; ISSN: 2610-802X

Universidad Nacional Experimental Francisco de Miranda (UNEFM). Santa Ana de Coro. Venezuela

Henry David Vásconez Samaniego; Victor Hugo Vásconez Samaniego; Luís Fernando Neira Altamirano; Francisca Fernanda Guerrero Chávez

DOI $10.35381 / \mathrm{cm} . v 5 \mathrm{i} 8.216$

\title{
Efectividad de un programa de capacitación gerencial - administrativa con la finalidad de consolidar las microempresas
}

\section{Effectiveness of a management training program - administrative in order to consolidate microenterprises}

\author{
Henry David Vásconez Vásconez \\ hdvasconezv@hotmail.com \\ Escuela Superior Politécnica de Chimborazo, Riobamba \\ Ecuador \\ https://orcid.org/0000-0003-2648-3445 \\ Víctor Hugo Vásconez Samaniego \\ vvasconez@unach.edu.ec \\ Universidad Nacional de Chimborazo, Riobamba \\ Ecuador \\ https://orcid.org/0000-0003-2997-4768 \\ Luís Fernando Neira Altamirano \\ luis.neira@unach.edu.ec \\ Universidad Nacional de Chimborazo, Riobamba \\ Ecuador \\ https://orcid.org/0000-0002-4147-2501 \\ Francisca Fernanda Guerrero Chávez \\ amantistafg@hotmail.com \\ Universidad Nacional de Chimborazo, Riobamba \\ Ecuador \\ https://orcid.org/0000-0002-2909-1271
}

Recibido: 26 de abril de 2019

Aprobado: 30 de mayo de 2019

\section{RESUMEN}

Se buscó determinar la en el Ecuador como alternativa viable para el buen vivir. La investigación fue de tipo explicativa con un diseño cuasi experimental de pre y pos test. 
El tratamiento aplicado al grupo experimental surtió efectos positivos por cuanto existió diferencia estadística entre el momento inicial (sin tratamiento) y el momento final (una vez aplicado el tratamiento), se rechaza $\mathrm{HO}$ y se acepta $\mathrm{H} 1$, lo que indica que el tratamiento aplicado fue favorable. La efectividad del programa desarrollado, permite contar con un grupo de micro empresarios en conocimiento de la importancia de estar actualizado en temas relacionados a la administración y gerencia, de ese modo, se puede articular el posicionamiento de una economía basada en lo sostenible y sustentable.

Descriptores: Administración de empresas; Microeconomía; Oferta y demanda; empresa privada.

\begin{abstract}
It was sought to determine the one in Ecuador as a viable alternative for good living. The research was explanatory with a quasi-experimental design of pre and post test. The treatment applied to the experimental group had positive effects because there was a statistical difference between the initial moment (without treatment) and the final moment (once the treatment was applied), $\mathrm{H} 0$ is rejected and $\mathrm{H} 1$ is accepted, indicating that the treatment applied was favorable. The effectiveness of the program developed, allows to have a group of micro entrepreneurs in knowledge of the importance of being updated on issues related to administration and management, in this way, the positioning of an economy based on sustainable and sustainable can be articulated.
\end{abstract}

Descriptors: Business management; Microeconomics; Supply and demand; Private enterprises.

\title{
INTRODUCCIÓN
}

Las empresas transcienden cuando son gerenciadas en capacidad de mantener la exigencia de la oferta y demanda, siendo pertinente contar con personas formadas para ser empresarios emprendedores, innovadores, en razón de estar en concordancia con las diversas expectativas y perspectivas de los clientes, Pallares y otros, (2002) definen al empresario como "una persona, entidad o grupo de personas que independientemente o asociados por voluntad propia y motivaciones individuales, deciden asumir un riesgo en la realización de un actividad económica determinadas y en la cual aspiran a tener éxito". (p.132), esto brinda la posibilidad de contar con diversas tipologías de empresa, siendo una de ellas, las microempresas o pequeñas empresas, operacionalizadas por 
emprendedores con la intención de generar bienes y servicios de calidad en razón del buen vivir.

La microempresa es concebida por Márquez y Portela (2002), como "un grupo de unidades productivas relativamente eficientes en la producción de bienes y servicios, estos operan en sector muy abiertos a la competencia, pero que cuentan con poco capital y con muy pequeñas en tamaño" (p. 42), esto posibilita la necesidad de capacitar a los microempresarios con la finalidad de fomentar mayores posibilidades de éxito y crecimiento, lo cual contribuye al fortalecimiento del sistema económico del Ecuador, produciendo muevas plazas de empleo, lo cual progresivamente hace crecer la calidad de vida de las personas.

Los emprendedores y microempresarios se basan sobre el norte de gestión de la empresa, Arieu (2003), expresa que el microempresario "es un productor de valores de mercado, que corre riesgos, está en permanente alerta para descubrir las oportunidades de ganancias que aún no han sido descubiertas y actúa en consecuencia para aprovecharlas" (p.8), lo planteado, se constituye en uno de los factores para triunfar empresarialmente, tanto a nivel micro como macro, un paso importante para tener acceso al conocimiento o aprendizaje es disponer de información sobre los alcances y los medios de la capacitación.

Por otro lado, Sánchez (2009), define la capacitación como "una herramienta básica para lograr mayor control de la administración de los recursos de las microempresas" (p.28), a pesar de la importancia de la misma para viabilizar la estabilidad de la microempresa en atención a la calidad en la prestación de bienes y servicios de la microempresa, se evidencia a través de la observación directa, la carencia de capacitación debilitando el crecimiento y desarrollo de estas en el tiempo. Así mismo, al desconocimiento del entorno en cuanto a los cambios de gusto, servicios y requerimientos como a los cambios tecnológicos, alcanzando un letargo intelectual.

Así mismo, Lefcovich (2010), plantea que "quienes crean microempresas lo hacen desconociendo las escasas probabilidades de supervivencia o a pesar de ella. La 
experiencia demuestra que el $50 \%$ de estas quiebran durante el primer año de actividad, y no menos del $90 \%$ antes de cinco años" (p.5), por lo tanto, el principal capital de la microempresa es contar con un personal formado integralmente para el abordaje efectivo de la gerencia de la organización, por consiguiente una microempresa será más eficiente, cuando más elevado sea la preparación y la calidad de sus empleados. En complemento, Lefcovich (2010), explica que la no aplicación de un plan de capacitación para la consolidación de los microempresarios, podría afectar del siguiente modo:

(a) Falta de Experiencia: Constituye en sí la base fundamental de todas las demás causas que llevan al fracaso, es necesario contar con experiencia en el ramo en particular a la cual se dedique. (b) Fallas en procesos interno: Altos niveles de deficiencia en materia de calidad y productividad, si no están acorde con los niveles del mercado, llevará a elevados costos de pedida de clientes. (c) Fallas para resolver problemas y tomar decisiones: La falta de detección y definición de problemas y sus causas, la incapacidad para generar soluciones factibles, y capacidad para su puesta en marcha, lleva a la no solución de los problemas a tiempo. (d) Mala gestión financiera: No tener el control sobre los ingresos y egresos. (e) Tener una mala actitud: No poseer actitud de lucha y sacrificio, aunado a una clara disciplina y ética de trabajo impedirá el crecimiento de la microempresa. (P.15-16).

Es urgente la aplicación de planes de capacitación que permitan a los microempresarios del Ecuador, consolidarse por cuanto representan un alto porcentaje de posibilidad del progreso económico de la nación, Blomberg (2018), alude que:

El negocio es parte de las denominadas mipymes (micro, pequeñas y medianas empresas) que cada 27 de junio celebran su día internacional. En Ecuador estos tres sectores representan el 99,55\%, según cifras del Instituto Nacional de Estadística y Censos (INEC) del 2017 (p. 1).

El casi $100 \%$ de la producción empresarial se ve representada por pequeñas empresas, por consiguiente se justifica la capacitación permanente de este sector, de ese modo, se podrá contar con posibilidad de fomentar una economía basada en el esfuerzo del pequeño productor, siendo necesario apoyarle en créditos, tecnología, pero sobre todo 
en capacitación gerencial - administrativa que les permita desarrollar un liderazgo asertivo con la finalidad de generar transformaciones socio económico en beneficio de la sociedad ecuatoriana, por consiguiente, la actual investigación tiene por objetivo determinar la efectividad de un programa de capacitación gerencial - administrativa con la finalidad de consolidar las microempresas en el Ecuador como alternativa viable para el buen vivir.

\section{DESARROLLO}

\section{Microempresas}

La microempresas es definida por Martínez (2006), como:

Comercios muy pequeños administrados por sus propietarios, pocos empleados. Con frecuencia, operan desde una residencia y tienen poco capital. Se transforman con facilidad por no tener una estructura rígida, adaptando sus productos a los cambios del mercado, son generadoras de empleo (p.76).

Por lo tanto, para que las microempresas logren el éxito obedece en buena parte a contar con el apoyo pertinente por parte de los entes públicos y privados de gestionar el comercio y la economía del país, siendo significativo aportar en la consolidación de una sociedad con base económica sustentable para su progreso. Por otro lado, Pérez (2003), afirma:

Las microempresas constituyen un medio económico que permite a sus miembros asumir directamente sus ingresos económicos para responder a las necesidades y aspiraciones; contribuyendo al desarrollo socioeconómico personal y extendiéndose a su comunidad, ya que esto abre oportunidades de trabajo a su entorno (p.53).

Al ser las microempresas un sector basado en la iniciativa de pequeños comerciantes o emprendedores con la finalidad de satisfacer sus necesidades económicas, en pertinencia de apoyar en la transcendencia de otras personas, se requiere de puntual capacitación que les permite auto administrar con eficiencia, los ingresos percibidos, siendo necesario que conozcan y manejen la planificación por cuanto le evitará caer en 
improvisaciones que afecten el efectivo desenvolvimiento de sus operaciones, Gómez (2007), indica que la planificación:

Es un proceso mediante el cual una organización define su negocio, la visión de largo plazo, y las estrategias para alcanzarlas, con base en el análisis de sus fortalezas, debilidades, oportunidades y amenazas. El mismo autor defines ese proceso como: Fortalezas: Son las actividades y los atributos internos de una organización que contribuyen y apoyan al logro de los objetivos de una institución. Debilidades: Son las actividades o tributos internos de una organización que inhiben o dificultan el éxito de una empresa. Oportunidades: Son los eventos, hechos o tendencias en el entorno de una organización que podrían facilitar o beneficiar el desarrollo de ésta, si se aprovecha en forma oportuna y adecuada. Amenazas: Son los eventos, hechos o tendencias en el entorno de una organización que inhibe, limita o dificulta su desarrollo operativo (p.53)

Por lo tanto, toda organización micro, pequeña o grande debe explorar el entorno interno y externo, para tomar previsiones en cuanto a debilidades, amenazas, así como aprovechar las oportunidades y las fortalezas que se le presente. Es importante fundamentar, que conocer las demandas o necesidades de los consumidores, así como la capacidad para cubrirlas, aunado a evaluar los cambios en las potencialidades de los clientes, proveedores y competidores, Francés (2001), complementa diciendo que la planificación "Ayuda a ajustarse a los cambios antes de que ocurran, soluciona los problemas que pueden ocurrir y permite disponer de tiempo para desarrollar los planes de acción" (p.26), siendo necesario contar dentro de la planificación empresarial con procesos de capacitación, Estrada (2003), expresa que:

Los planes de capacitación ayudan a las personas a desempeñar su trabajo actual y los beneficios de ésta pueden extenderse a toda su vida laboral o profesional, además pueden ayudarlos a desarrollar habilidades para responsabilidades futuras, dichos planes cuenta con objetivos muy claros, entre los cuales se pueden mencionar: Conducir a la empresa a una mayor rentabilidad y a los empleados a tener una actitud más positiva, mejorando el conocimiento del puesto a todos los niveles. Ayudando al personal a identificarse con los objetivos de la empresa, fomentando la autenticidad, la apertura y la confianza (p. 210). 
Universidad Nacional Experimental Francisco de Miranda (UNEFM). Santa Ana de Coro. Venezuela

Henry David Vásconez Samaniego; Victor Hugo Vásconez Samaniego; Luís Fernando Neira Altamirano; Francisca

Se indica que la capacitación permite generar la posibilidad de promover la calidad y éxito de las microempresarios en razón de responder a sus necesidades, como a las perspectivas - expectativas del cliente, en complemento Estrada (2003), indica que:

Los beneficios de la capacitación no son sólo para el trabajador, sino también para la empresa; ya que para ambos constituye la mejor inversión para enfrentar los retos del futuro. Destacando beneficios como: un trabajo preparado para el manejo de conflictos, la toma de decisiones y la solución de problemas mediante herramientas técnicamente probadas; aumentando así la confianza del individuo. Lo que permite a su vez aumentar las metas individuales dentro de la organización y elevar el nivel de satisfacción en el cargo. Otro elemento de suma importancia es la mejora de la comunicación intrainstitucional e interinstitucional; a través de los planes de capacitación, contribuyendo a la integración grupal dentro de la empresa y a una mejor relación con el entorno (p.230)

Se manifiesta que una organización que aprende a aprender es aquella que transfiere conocimientos a sus miembros, que construye un capital que no sólo se refleja en las cuentas de resultados de las empresas sino también en el potencial de sus miembros. Chiavenato (2007), establece que un plan de capacitación es un "Conjunto de estrategias, actividades, recursos necesarios para aumentar el desempeño y rendimiento de las microempresas a través de la mejora de sus conocimientos, habilidades y actitudes, de acuerdo a sus necesidades" (p. 98).

La capacitación debe tratar de orientar experiencias de aprendizaje hacia lo positivo, complementarlas y reforzarlas con actividades planeadas para que los individuos en todos los niveles de la organización puedan adquirir conocimientos con mayor rapidez con la finalidad de desarrollar aquellas actitudes y habilidades que los beneficiarían a sí mismos, y a su empresa. Por consiguiente, cubre una secuencia programada de eventos que pueden expresarse como un proceso continuo cuyo ciclo se renueva cada vez que se repite, con el propósito de generar en los empleados habilidades y conductas que le permitan ser un sujeto proactivo, capaz de tomar iniciativas tomando en cuenta el aprendizaje adquirido. Así mismo, Chiavenato (2007), señale que para lograr el objetivo 
anterior el contenido de la capacitación puede incluir cuatro formas de cambios de conductas a saber:

1. Transmisión de información: es el elemento esencial de muchos programas de capacitación, es decir, la información que se imparte entre los educandos en forma de un conjunto de conocimientos. Normalmente, la información es general, preferentemente sobre el trabajo, como información respecto a la organización, sus productos y servicios, sus estructuras y políticas.

2. Desarrollo de habilidades: Sobre todo las habilidades, las destrezas y los conocimientos que están directamente relacionados con el desempeño del puesto presente o de posibles funciones futuras. Se trata de una capacitación orientada directamente hacia las tareas y las operaciones que serán realizadas.

3. Desarrollo o modificación de actitudes: se busca generalmente la modificación de actitudes negativas de los trabajos para convertirlas en otras más favorables, como aumentar la motivación o desarrollar la sensibilidad del personal de gerencia y de supervisión en cuanto a los sentimientos y las reacciones de las personas.

4. Desarrollo de conceptos: La capacitación puede estar dirigida a elevar la capacidad de abstracción y la concepción de ideas y filosofías, sea para facilitar la aplicación de conceptos en la práctica de la administración, sea para elevar el nivel de generalización para desarrollar gerentes que puedan pensar en términos globales y amplios. (p. 265)

En este sentido, un plan de capacitación al sector micro empresarial debe estar concebido desde su realidad en función de brindar oportunidad de crecimiento integral a los empresarios y quienes laboran con él, de ese modo, existe la posibilidad real de que se generen cambios en la estructura organizativa que les permita promover un trabajo efectivo en razón de estar a la altura de las exigencias socio económicas de un mercado global y voraz que procura aniquilar la 
CIENCIAMATRIA

Revista Interdisciplinaria de Humanidades, Educación, Ciencia y Tecnología

Año V. Vol. V. N09. Julio - Diciembre 2019

Hecho el depósito de ley: pp201602FA4721

ISSN-L: 2542-3029; ISSN: 2610-802X

Universidad Nacional Experimental Francisco de Miranda (UNEFM). Santa Ana de Coro. Venezuela

Henry David Vásconez Samaniego; Victor Hugo Vásconez Samaniego; Luís Fernando Neira Altamirano; Francisca

Fernanda Guerrero Chávez

competencia, siendo esto una amenaza para las empresas que no le brinden la debida importancia a la capacitación gerencial - administrativa.

\section{METODOLOGÍA DE LA INVESTIGACIÓN}

La investigación fue de tipo explicativa, Hernández Sampieri, Fernández Collado \& Baptista (2014), la definen "como aquellos estudios que van más allá de la descripción de los fenómenos". Para el análisis estadístico del pre y post test se aplicó la prueba T de Student. Construyéndose las siguientes hipótesis: Nula H0: No existe diferencias significantes entre ambos momentos, lo que es igual no funcionó el tratamiento. Alterna $\mathrm{H} 1$ : Existen diferencias significantes entre ambos momentos, lo que es igual, sí funcionó el tratamiento. La población objeto de estudio estuvo conformada por 31 microempresarios de la ciudad de Manta, la muestra que participó en el grupo experimental (tratamiento) fue de 18 personas quienes conformaron el grupo RG1, mientras que otros 13 conformaron el grupo control o RG2 (no se les aplicó tratamiento).

\section{RESULTADOS DE LA INVESTIGACIÓN}

Se procedió a aplicar una prueba de hipótesis utilizando un nivel de significancia de $5 \%$. Las hipótesis planteadas son:

Nula HO: No existe diferencias significantes entre ambos grupos, lo que es igual no funcionó el tratamiento.

Alterna H1: Existen diferencias significantes entre ambos grupos, lo que es igual, sí funcionó el tratamiento. 
CIENCIAMATRIA

Revista Interdisciplinaria de Humanidades, Educación, Ciencia y Tecnología

Año V. Vol. V. N09. Julio - Diciembre 2019

Hecho el depósito de ley: pp201602FA4721

ISSN-L: 2542-3029; ISSN: 2610-802X

Universidad Nacional Experimental Francisco de Miranda (UNEFM). Santa Ana de Coro. Venezuela

Henry David Vásconez Samaniego; Victor Hugo Vásconez Samaniego; Luís Fernando Neira Altamirano; Francisca Fernanda Guerrero Chávez

Prueba T de Student a partir de aplicación de encuesta.

Figura 1.

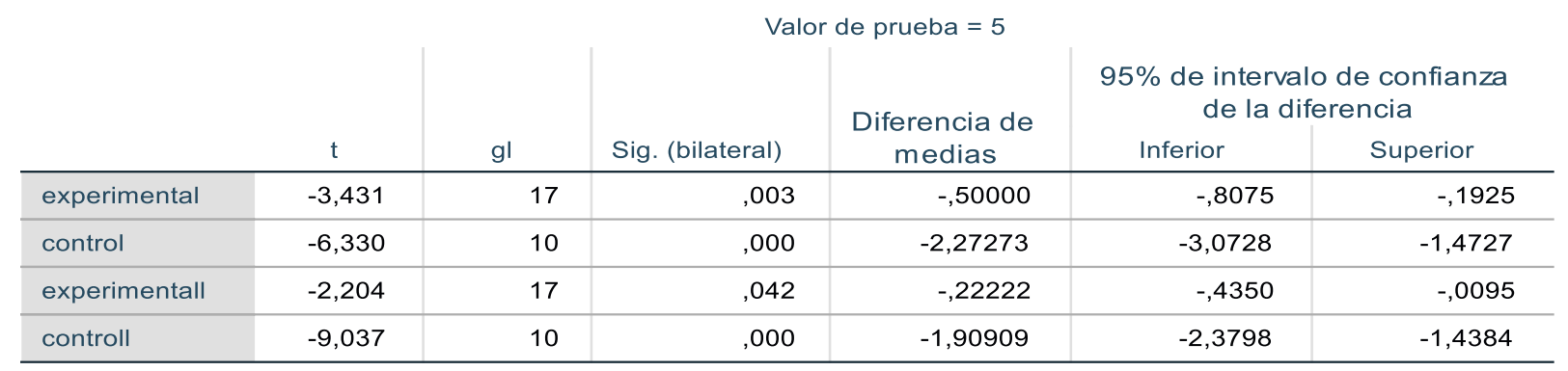

Al realizar la prueba $T$ de Student para muestras independientes, se tiene que el grupo experimental en el pre test presentó significancia de 003, mientras que el grupo control ,000. En el pos test el grupo experimental generó significancia de 0,42 y el grupo control , 000

Al compararse ambos momentos se tiene que existe diferencia de significancia del grupo experimental entre el pre y pos test, mientras que el grupo control no evidenció cambios en ambos momentos, lo que permite indicar que el grupo experimental tuvo modificaciones conceptuales sobre el tratamiento aplicado, lo cual indica que el mismo resulto significante. En este sentido, se procedió a aceptar la hipótesis alterna:

Alterna H1: Existen diferencias significantes entre ambos grupos, lo que es igual, sí funcionó el tratamiento.

El tratamiento aplicado al grupo experimental surtió efectos positivos por cuanto existió diferencia estadística entre el momento inicial (sin tratamiento) y el momento final (una vez aplicado el tratamiento), se rechaza $\mathrm{HO}$ y se acepta $\mathrm{H} 1$, lo que indica que el tratamiento aplicado fue favorable.

\section{CONCLUSIONES}

El tratamiento aplicado, consistente en un programa de capacitación basado en crecimiento personal, microempresas, marketing, administración y contabilidad básica de empresas, liderazgo, comunicación, planificación estratégica, relación con entidades 
bancarias, visión ecológica y sustentable de la empresa, de ese modo se procedió a generar un plan integral que permitió abordar diversos factores relacionados a la gestión empresarial en aras de consolidar el sector de la micro empresa.

La efectividad del programa desarrollado, permite contar con un grupo de micro empresarios en conocimiento de la importancia de estar actualizado en temas relacionados a la administración y gerencia, de ese modo, se puede articular el posicionamiento de una economía basada en lo sostenible y sustentable, siendo importante destacar del plan de capacitación el enfoque de marketing ecológico por cuanto esto permite generar una visión ciudadana acorde a los retos globales 2030, siendo esto importante en el empresario responsable y con visión global ecológica, tal como planten Aldana Zavala\& Colina Ysea (2019).

Por otro lado, León (2017), señala la importancia de generar transformaciones en las organizaciones empresariales mediante la formación permanente de sus miembros, Aldana, Salón\& Guzmán (2019), esto posibilita la generación de un liderazgo sistémico que permita operar la empresa con visión sistémica desde la óptica de las organizaciones inteligentes, lo cual permite generar la visión de aprender continuamente y de modo permanente.

\section{REFERENCIAS CONSULTADAS}

1. Aldana Zavala, J., \& Colina Ysea, F. (2019). Marketing verde en la conformación de una ciudadanía planetaria en el ámbito educativo latinoamericano. Revista San Gregorio, O(31), 150-161. doi:http://dx.doi.org/10.36097/rsan.v0i31.972

2. Aldana, J., Salón, M., \& Guzmán, N. (2019). Liderazgo sistémico en las competencias gerenciales docentes universitarias. CIENCIAMATRIA, 5(8), 50-74. Recuperado a partir de http://cienciamatriarevista.org.ve/index.php/cm/article/view/87

3. Arieu A. (2003). Negocios exitosos en cinco pasos. Artículo "Apoyo a Emprendedores" http://winred.com/emprender.com 
4. Blomberg, C. (2018). Las mipymes representan el $99 \%$ de negocios en Ecuador. Recuperado https://www.eluniverso.com/noticias/2019/06/27/nota/7396308/mipymesrepresentan-99-negocios-pais

5. Chiavenato, I. (2007). Administración de Recursos Humanos, El Capital Humano de las Organizaciones. Octava Edición. Colombia: Editorial McGraw-Hill.

6. Estrada, S. (2003). Formación, Capacitación, Desarrollo de RR.HH y su importancia en las Organizaciones. México McGraw-Hill.

7. Francés A. (2001). Estrategias para la empresa en América Latina. Producción Editorial, Ediciones IESA, C.A.

8. Gómez H. (2007). Gerencia Estratégica. Novena Edición, Editorial: 3R Editores, Bogotá

9. Hernández Sampieri, R., Fernández Collado , C., \& Baptista , L. (2014). Metodología de la investigación . México: Editorial Mc - Graw - Hill Interamericana. Sexta edición.

10. León, F. (2017). Transposición de saberes desde el análisis organizacional de las metáforas de Morgan: herramienta para romper paradigmas de la gerencia académica universitaria en Venezuela. Revista Arbitrada Interdisciplinaria Koinonía, 2(3), 31-47. Recuperado de http://fundacionkoinonia.com.ve/ojs/index.php/revistakoinonia/article/view/50/37

11. Lefcovich, M. (2010). Las pequeñas empresas y causas de sus fracasos. Recuperado de http://www.degerencia.com/mlefcovich

12. Martínez A. (2006). Microempresa y pobreza en Venezuela: un caso de estudio. Revista Mexicana de Ciencias Políticas y Sociales.

Año XLVIII Número. 198 septiembre- diciembre de 2006.

13. Marques G. y Portela C. (1990). Los Informales Urbanos en Venezuela: ¿pobres o eficientes? Primera edición. Universidad de Texas.

14.Pallares Z. Romero D. y Herrera M. (2002). Hacer Empresa: un reto. Tercera edición. Bogotá, Editorial McGraw-Hill.

15. Pérez V. (2003). Las Micro finanzas: el Inicio de la estructura intermedia para 
Revista Interdisciplinaria de Humanidades, Educación, Ciencia y Tecnología

Año V. Vol. V. N09. Julio - Diciembre 2019

Hecho el depósito de ley: pp201602FA4721

ISSN-L: 2542-3029; ISSN: 2610-802X

Universidad Nacional Experimental Francisco de Miranda (UNEFM). Santa Ana de Coro. Venezuela

Henry David Vásconez Samaniego; Victor Hugo Vásconez Samaniego; Luís Fernando Neira Altamirano; Francisca Fernanda Guerrero Chávez

Venezuela. Visión General. ISSN: 1317-8822, año 2, número 1, volumen 1 enero - junio 2003.

16. Sánchez D. (2009). Entrevista a D. Antonio Sánchez. Recuperado de www.diariojurídico.com

\section{REFERENCES CONSULTED}

1. Aldana Zavala, J., \& Colina Ysea, F. (2019). Green marketing in the conformation of a planetary citizenship in the Latin American educational field. San Gregorio Magazine, 0 (31), 150-161. doi: http: //dx.doi.org/10.36097/rsan.v0i31.972

2. Aldana, J., Salón, M., \& Guzmán, N. (2019). Systemic leadership in university teaching management skills. SCIENCE, 5 (8), 50-74. Recovered from http://cienciamatriarevista.org.ve/index.php/cm/article/view/87

3. Arieu A. (2003). Successful business in five steps. Article "Support for Entrepreneurs" http://winred.com/emprender.com

4. Blomberg, C. (2018). MSMEs represent $99 \%$ of businesses in Ecuador. Recovered from https://www.eluniverso.com/noticias/2019/06/27/nota/7396308/mipymesrepresentan-99-negocios-pais

5. Chiavenato, I. (2007). Human Resources Administration, The Human Capital of Organizations. Eighth edition. Colombia: McGraw-Hill Publishing.

6. Estrada, S. (2003). Training, Training, Human Resources Development and its importance in Organizations. Mexico McGraw-Hill.

7. French A. (2001). Strategies for the company in Latin America. Editorial Production, Ediciones IESA, C.A.

8. Gómez H. (2007). Strategic management. Ninth Edition, Editorial: 3R Editores, Bogotá

9. Hernández Sampieri, R., Fernández Collado, C., \& Baptista, L. (2014). Investigation methodology . Mexico: Editorial Mc - Graw - Interamerican Hill. Sixth edition.

10. León, F. (2017). Transposition of knowledge from the organizational analysis of Morgan's metaphors: a tool to break paradigms of university academic management in Venezuela. Interdisciplinary Arbitrated Review Koinonía, 2 (3), 31 - 


\section{CIENCIAMATRIA}

Revista Interdisciplinaria de Humanidades, Educación, Ciencia y Tecnología

Año V. Vol. V. N09. Julio - Diciembre 2019

Hecho el depósito de ley: pp201602FA4721

ISSN-L: 2542-3029; ISSN: 2610-802X

Universidad Nacional Experimental Francisco de Miranda (UNEFM). Santa Ana de Coro. Venezuela

Henry David Vásconez Samaniego; Victor Hugo Vásconez Samaniego; Luís Fernando Neira Altamirano; Francisca Fernanda Guerrero Chávez

47.

Recovered from

http://fundacionkoinonia.com.ve/ojs/index.php/revistakoinonia/article/view/50/37

11. Lefcovich, M. (2010). Small businesses and causes of their failures. Recovered from http://www.degerencia.com/mlefcovich

12. Martínez A. (2006). Microenterprise and poverty in Venezuela: a case study. Mexican Journal of Political and Social Sciences. Year XLVIII Number. September 198- December 2006.

13. Marques G. and Portela C. (1990). Urban Informals in Venezuela: poor or efficient? First edition. University of Texas

14. Pallares Z. Romero D. and Herrera M. (2002). Doing Business: a challenge. Third edition. Bogotá, McGraw-Hill Publishing.

15.Pérez V. (2003). Micro Finance: the Beginning of the intermediate structure for Venezuela. Overview. ISSN: 1317-8822, year 2, number 1, volume January 1 June 2003.

16.Sánchez D. (2009). Interview with Mr. Antonio Sánchez. Recovered from www.diariojurídico.com

C2019 por los autores. Este artículo es de acceso abierto y distribuido según los términos y condiciones de la licencia Creative Commons Atribución-NoComercial-Compartirlgual 4.0 Internacional (CC BY-NC-SA 4.0)

(https://creativecommons.org/licenses/by-nc-sa/4.0/). 